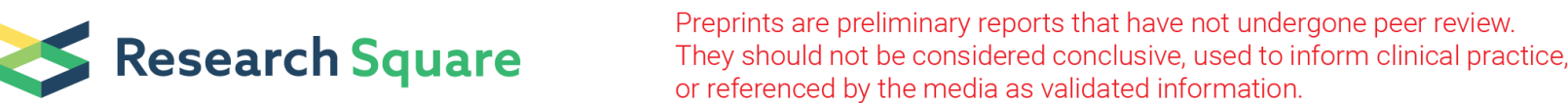

\section{Immediate and 6-Week Effects of Wearing a Knee Sleeve Following Anterior Cruciate Ligament Reconstruction on Knee Kinematics and Kinetics: A Cross-Over Laboratory and Randomised Clinical Trial}

Gisela Sole ( $\square$ gisela.sole@otago.ac.nz )

University of Otago

Peter Lamb

University of Otago

Todd Pataky

Kyoto University

Anupa Pathak

University of Sydney

Stefan Klima

Orthopaedicus Leipzig

Pierre Navarre

Southland Hospital

Niels Hammer

Medical University of Graz

\section{Research Article}

Keywords: Anterior cruciate ligament reconstruction, hopping, kinematics, kinetics, flexion angle trajectory, knee sleeve

Posted Date: February 18th, 2022

DOI: https://doi.org/10.21203/rs.3.rs-1326038/v1

License: (c) (i) This work is licensed under a Creative Commons Attribution 4.0 International License.

Read Full License 


\section{Abstract}

\section{Background}

Elastic knee sleeves are often worn following anterior cruciate ligament reconstruction (ACLR) but their effects on movement patterns are unclear.

\section{Aim}

To determine the immediate and 6-week effects of wearing a knee sleeve on biomechanics of the knee during a step-down hop task.

\section{Methods}

Using a cross-over design, we estimated sagittal plane knee kinematics and kinetics and stance duration during a step-down hop for 31 participants (age 26.0 [SD 6.6] years, 15 women) after ACLR (median 21 months post-surgery) with and without wearing a knee sleeve. In a subsequent randomised clinical trial, participants in the 'Sleeve Group' $(n=9)$ then wore the sleeve for 6 weeks at least 1 hour daily, while a 'Control Group' ( $\mathrm{n}=9$ ) did not wear the sleeve. We used statistical parametric mapping to compare (1) knee flexion/extension angle and external flexion/extension moment trajectories between three conditions at baseline (uninjured side, unsleeved injured side and sleeved injured side); (2) withinparticipant changes for knee flexion angles and external flexion/extension moment trajectories from baseline to follow-up between groups. We compared discrete flexion angles and moments, and stance duration between conditions and between groups.

\section{Results}

Without sleeves, knee flexion was lower for the injured than the uninjured sides during mid-stance phase. When wearing the sleeve on the injured side, knee flexion increased during the loading phase of the stance phase. Discrete initial and peak knee flexion angles increased by (mean difference, $95 \% \mathrm{Cls}$ ) $2.7^{\circ}$ $(1.3,4.1)$ and $3.0^{\circ}(1.2,4.9)$, respectively, when wearing the knee sleeve. Knee external flexion moments for the unsleeved injured sides were lower than the uninjured sides for $80 \%$ of stance phase, with no change when sleeved. The groups differenced for within-group changes in knee flexion trajectories at follow-up. Discrete knee flexion angles increased for the Control group only. Stance duration decreased by $22 \%$ for the Sleeve group from baseline to follow-up $(-89 \mathrm{~ms} ;-153,-24)$ but not for the Controls.

\section{Conclusions}

Application of knee sleeves following ACLR is associated with improved knee flexion angles during hop landing training. Longer term (daily) knee sleeve application may help improve hop stance duration, indicating improved hop performance.

\section{Trial Registration}


The trial was prospectively registered with the Australia New Zealand Clinical Trials Registry No: ACTRN12618001083280, 28/06/2018

https://anzctr.org.au/Trial/Registration/TrialReview.aspx?id=375347\&isClinicalTrial=False

\section{Introduction}

Rehabilitation following anterior cruciate ligament $(A C L)$ reconstruction includes progressive exercise prescription to improve range of motion, muscle strength, sensori-motor control and sports- and workspecific skills, as well as physical fitness [1]. Psychosocial factors, such as fear of re-injury, are also considered and strategies are included to improve confidence and self-efficacy for return to physical activity $[2,3]$. Such strategies may include prescription of wearing a knee sleeve, or people with ACL reconstruction may intuitively use them $[2,4]$. Wearing a knee sleeve appears to influence sensorimotor control, potentially leading to immediate enhanced movement patterns [6-8].

Jump-landing strategies have received substantial attention as a risk factor for ACL rupture and as outcomes following such injury $[9,10]$. In general, landing with less hip, knee and ankle flexion, thus a more 'extended knee' is considered to increase risk of re-injury, or sub-optimal recovery following injury [11]. Various interventions have been explored to improve lower limb biomechanics during jump-landing, including plyometric training with or without verbal and augmented feedback $[10,12,13]$.

A systematic review suggested that jump landing with augmented feedback (visual display, sound and/or vibration) was effective in immediate increased knee flexion angles and vertical ground reaction forces during jump landing [10]. Differences for knee flexion moments, however, were not significant [10]. Less is known for long-term effects of interventions for landing mechanics. One 4-week plyometric exercise programme did not improve jump landing mechanics in participants with ACL reconstruction [14].

We previously reported that wearing a knee sleeve, on average, led to an immediate improved distance of a single leg hop in participants with residual self-reported functional limitations following ACL reconstruction [5]. However, we found no significant differences over a six-week period between a smaller group of participants wearing the sleeve at least one hour daily compared to a control group. Similarly, no between-group differences were found for self-reported knee function (assessed with the International Knee Disability Committee - Short Knee Form, IKDC-SKF), and thigh muscle strength at follow-up [5].

The aim of this second study was to determine immediate and six-week effects of wearing a knee sleeve on knee kinematics and kinetics in participants with an ACL reconstruction in the previous 5 years, specifically who had residual self-reported functional limitations. The primary research hypothesis $(\mathrm{H} 1)$ was that knee flexion and knee external flexion moments would increase during the stance phase of the step-down-hop task when wearing a sleeve compared to not wearing the sleeve. Secondary hypotheses were that the group wearing the sleeve for a six-week period would have increased knee flexion angles and knee flexion moments during the stance phase than the control group ( $\mathrm{H} 2)$, and that stance duration of the step-down-hop would decrease while wearing the sleeve compared to not wearing the sleeve (H3). 


\section{Methods}

Data were collected during two sessions (baseline and six-week follow-up) in a university research laboratory and via REDCap (Research Electronic Data Capture, hosted by the University of Otago, Dunedin, New Zealand). Ethical approval was granted by the Health and Disability Ethics Committee (of New Zealand). We followed CONSORT reporting guidelines [15]. The sample of this study was the same sample as in the previous report [5].

\section{Trial design and binding}

Part 1 of the study consisted of a cross-over laboratory-based study, exploring immediate effects of wearing the knee sleeve. It was impossible to blind participants and assessors to the sleeve condition in Part 1. Part 2 was a parallel two-armed, assessor-blinded randomised clinical trial (RCT), with the same participants as in Part 1 [5]. The biostatistician and the research assistant were blinded to group allocation for the RCT.

\section{Participants}

\section{Recruitment}

We recruited participants via community advertising and using TrialFacts (https://trialfacts.com/), a research participant recruitment agency. Recruitment started in September 2018 and closed in September 2020 due to the end of the funding period. Volunteers completed a questionnaire (also serving as screening for eligibility) via REDCap prior to attending the first laboratory session [5]. The questionnaire included demographics, injury and surgery history, the International Knee Documentation Committee Subjective Knee Form (IKDC-SKF) [16] and the Tegner activity scale [17].

\section{Inclusion criteria}

We recruited men and women, aged 18-40 years, who underwent ACL reconstruction within 6 months to 5 years previously [5]. We targeted individuals who had not yet reached full functional level, for the purpose of this study defined by a score between 40 to $80 / 100$ on the IKDC-SKF $[16,18,19]$. The initial protocol defined a time period for an ACL reconstruction as 6 months to 3 years previously. Due to slow recruitment rate, and amendment was approved by the ethics committee to expand the period until 5 years post-ACL reconstruction.

\section{Exclusion criteria}

We excluded participants who had undergone a revision ACL reconstruction of the same knee (due to reinjury), or a previous ACL reconstruction of the opposite knee; self-reported any other lower limb, pelvic or low back musculoskeletal injuries or disorders that required medical care over the past 6 months; had known systemic, neurological or cardiovascular disorders; or had a body mass index (BMI) above 30 $\mathrm{kg} / \mathrm{m}^{2}$ [5]. We excluded participants with an IKDC-SKF score less than 40 (due to potential safety risk 
during the laboratory-based tasks) or greater than $80 / 100$ (as use of a sleeve would clinically be less likely to add benefit) [5].

\section{Procedures}

\section{Randomisation}

We randomised each participant twice (once for the cross-over trial, and once for the RCT) with equal numbers in each group for both allocations. The research officer sequentially block randomised groups of 8 participants with an electronic random number generator before participants entered the study. Each group was stratified by sex. The research officer informed the assessor for the laboratory data collection of the order for the conditions for the cross-over trial, and the group allocation (for the RCT) via email prior to the participant's first laboratory session [5]. Participants provided written informed consent at the start of the first session. Participants were dressed in a singlet, a pair of shorts and their own sport shoes. Body mass and height were measured during the baseline session [5].

\section{Part 1: Laboratory cross-over trial}

Participants undertook two hopping tasks: a maximum horizontal single leg hop and a sub-maximum step-down hop. A sub-maximal level was chosen for safety as we were seeking participants with remaining residual functional limitations (as defined by the IKDC-SKF). To allow comparison of knee mechanics across the six-week period, the distance of the required hop was standardised and individualised based on the participants single-leg hop distance of the uninjured side.

Participants practised the hopping tasks at sub-maximal distance with the uninjured and injured sides until they were confident with performing them as part of familiarisation and warm-up. They performed the maximum horizontal hop prior to undertaking the step-down-hop.

\section{Part 2 Randomised clinical trial}

On completion of the first laboratory session, the assessor informed participants of their group allocation for the RCT. All participants were asked to return to the laboratory following the 6-week period to repeat the above assessments, repeating the hopping tasks without wearing the knee sleeve [5].

\section{Intervention}

We used a commercially available knee sleeve, GenuTrain (Bauerfeind® AG, Zeulenroda-Triebes, Germany), a CE-certified medical device, as the intervention. The sleeve consisted of flexible elastic/knitted materials, providing knee support without limiting the range of motion [5]. All participants performed the step-down hop with and without the sleeve for the cross-over trial (Part 1). For Part 2 (RCT), only participants of the 'Sleeve Group' (intervention) were provided with the knee sleeve for the 6-week period. They were instructed to wear the sleeve for a minimum of 1 hour per day during their rehabilitative exercises, physical activity and sports. The control group were not provided with a sleeve during this period. As reported previously [5], participants of the 'Sleeve Group' were asked to document the use of 
the knee sleeve in a daily diary (Microsoft ${ }^{\circledR}$ Excel spreadsheet) and all participants were asked to document their daily physical activity and exercise.

The researcher explained the use of the knee sleeve to the 'Sleeve Group' and provided them with an instructional leaflet. They were informed to discontinue use of the knee sleeve and contact the researcher should any side-effects evolve, such as discomfort during use, pain, burning sensations of the knee, leg or foot, are swelling of the knee or calf [5].

\section{Outcomes}

1) Single-leg horizontal jump: After familiarisation with the tasks, they performed 3 trials of single-leg maximum horizontal jump, as described in the first report [5]. The maximum jump distance of the uninjured side during Session 1 was used to calculate the individualised target for the hop distance during the step-down task. The target step-down hop distance was $60-70 \%$ of the maximum single leg hop distance of the uninjured side, and was kept constant for the participant across the two sessions.

2) Step-down hop: Three-dimensional motion analysis was performed for the step-down hop with 11 infra-red cameras (Motion Analysis Corporation, Santa Rosa, CA, USA), sampling at $120 \mathrm{~Hz}$, and Cortex 2.0 software, synchronized with a floor-mounted force plates (BP2436 AMTI Inc., Newton, MA, USA), sampling at 2,400 Hz. A set of 42 reflective markers (diameter $12.5 \mathrm{~mm}$ ) were applied to the trunk, pelvis and lower extremities (Fig. 1). The marker positions were tracked by the camera system, reconstructed in 3D space and used to define segment coordinate systems.

Following the marker placement, a static anatomical calibration trial was performed with the participants standing still, on both feet. A functional movement trial was then performed by moving the hip in the three planes for calculation of functional hip joint centres [20]. One trial was undertaken for each side respectively.

The participants were then asked to stand on a 30-cm box, placed $15 \mathrm{~cm}$ from the force plate, and performed a step-down hop (adapted from Kristianslund and Krosshaug [21]) onto the force plate: the participant was asked to step off the box with either the injured or the uninjured leg onto the force plate (Fig. 2A), then hop forward off the plate as fast as possible. The distance of that hop was $60-70 \%$ of the maximum horizontal jump distance of the uninjured side (Fig. 2B). They performed the step-down-hop with the uninjured side first, then the injured side under the (1) 'control' condition (no sleeve) and (2) the 'sleeve' condition (experimental, wearing the sleeve), ordered by randomisation. A 5-minute walk between the conditions provided a standardised run-in to the second condition to minimise carryover effects.

\section{Data processing and analysis}

Cortex 5.5 (Motion Analysis Corporation, Santa Rosa, CA, USA) was used to track and label the markers, and the biomechanical model, kinematic (joint angles) and kinetic (moments) data were calculated using Visual3D Professional v6 (C-Motion, Inc., Germantown, MD, USA). Functional hip joint centres were 
estimated based on a movement trial involving all degrees of freedom of the hip [22], while anatomical joint centres at the knee and ankle joints were based on medial and lateral markers [20]. Marker clusters attached to a rigid base were fixed to the thighs and shanks to minimise marker coordinate estimation error [23]. Kinematic and kinetic data were filtered using a low-pass, double, second order Butterworth filter with a cut-off of $10 \mathrm{~Hz}$.

Data from the stance phase of the hop were of interest; the start and end of the stance phase were defined by the vertical component of the ground reaction force exceeding and returning below $20 \mathrm{~N}$, respectively. Peak knee flexion angles and flexion angles at initial contact were extracted for the stance phase. Based on the biomechanical model's segment inertial properties and joint properties, inverse dynamics analyses were applied to the model kinematics and ground reaction forces to calculate the net joint moments. Net joint moments were resolved into the proximal segment's coordinate system and represented as vectors. The moments were normalised to body size ( $\left.\mathrm{Nm} / \mathrm{BW}^{\star} \mathrm{HT}\right)$. The temporal variable was the duration of the stance phase of the step-down hop. For all variables, the averages of five trials for each limb (injured versus uninjured) and condition (sleeved and unsleeved) for each participant were calculated.

\section{Sample size}

We report a secondary analysis of a larger study investigating effects of wearing a knee sleeve for individuals following ACL reconstruction. The sample size was based on the primary outcome measure, the horizontal hop distance, as reported in [5]. We did not undertake a sample size calculation specifically for the outcomes reported in the current paper.

\section{Data analysis}

Demographic data were presented descriptively (means and standard deviations for approximately normally distributed continuous variables; medians and ranges for other continuous variables; and counts and percentages for categorical variables).

\section{Statistical parametric mapping}

We analysed kinetic and kinematic trajectories using Statistical Parametric Mapping (SPM, http://spm1d.org/; Pataky, 2012) [24]. SPM allows comprehensive statistical analyses of the trajectory of a given biomechanical variable (such as knee angles) during a specific task, using the entire $n$ dimensional biomechanical sampling data. That contrasts with discrete point analyses, such as peak knee flexion, that assess only the one point during the entire movement. SPM uses Random Field Theory to make probabilistic conclusions based on the random behaviour of that 1D observational unit. Scalar observations of discrete variables are based on traditional OD Gaussian randomness [25]. Thus, the whole trajectory can be assessed with SPM, and hypotheses relating to differences at specific time points during the task (such as a hop) do not need to be defined a priori. 
Stance phase kinematic and kinetic trajectories in the sagittal plane were temporally normalised to stance duration using linear interpolation across 100 equally spaced time points. Trajectories of five trials for each limb and each session were computed using MATLAB R2018b (The MathWorks Inc., Natick, MA, USA). The mean trajectory for the uninjured and injured sides (sleeved and unsleeved conditions, Session 1) were then computed.

For Part I, one-way repeated measures ANOVA was used to compare three conditions at the baseline: (a) uninjured side, unsleeved to (b) injured side, unsleeved, and (c) injured side, sleeved. ANOVA was conducted separately for each of two dependent variables: (i) knee flexion, and (ii) external flexion moment, and a conservative Bonferroni threshold of 0.025 was adopted to correct for multiple comparisons across these two tests. Post-hoc analyses using paired t tests and a secondary Bonferroni correction across the three pairwise tests $(a+b, a+c$ and $b+c)$ were used to assess between-condition effects.

For Part 2, within-participant changes were depicted between baseline and follow-up sessions (unsleeved only). An SPM independent $t$ test was used to compare within-participant changes in the Sleeve group versus the Control Group. This t test was repeated for each of the two aforementioned dependent variables, again with a conservative Bonferroni correction of 0.025 .

\section{Discrete variable analysis}

Post hoc analyses were also performed for the discrete variables. Analyses for Part 1 (cross-over trial) were adjusted for participant sex, surgery type, and time since surgery (as a continuous measure), and sequence effect. Analyses for the knee flexion angles (angle at initial contact, peak angle, and excursion), knee peak external flexion moment and stance duration are from linear mixed models using Restricted Maximum Likelihood (REML) to estimate random effects. A random participant effect and, where both limbs are measured at multiple time points, a random measurement occasion effect nested within participant. These analyses were performed with Stata (16.1, StataCorp LLC, College Station, TX, USA).

For Part 2 (RCT), individual change scores from baseline to follow-up were calculated for knee flexion angles, moments and stance duration. The change scores were compared between the Sleeve Group and the Control Group using Mann-Whitney U tests for each outcome. The alpha level was set at $p \leq 0.05$. These analyses and those of demographic data were performed with SPSS Version 24.0 (IBM Corp, Armonk, NY). Test-retest reliability from our laboratory for discrete variables of peak knee flexion, external peak flexion moment and stance duration are shown in the supplementary file.

\section{Results}

We assessed 34 participants at baseline, but data for three participants were excluded due to technical issues. Two participants of the Sleeve Group withdrew from the study following baseline assessment due to knee re-injuries, unrelated to use of the knee sleeve (Fig. 3). Eight participants were lost to follow-up due to the COVID-19 lockdown in New Zealand, March/April 2020. Twenty-four participants completed 
the follow-up laboratory session. Data from six participants was excluded due to technical difficulties, resulting in data being analysed for nine participants in each group for Part 1 (RCT). Demographic data of the participants are provided in Table 1.

Table 1

Demographic data $(n=31)$

\begin{tabular}{|llll|}
\hline & All & Men & Women \\
\hline Men/Women n (\%) & 31 & $16(52)$ & $15(48)$ \\
\hline Age (years) & $26.0(6.6)$ & $24.6(5.6)$ & $27.5(7.5)$ \\
\hline Mass $(\mathrm{kg})$ & $76.4(11.6)$ & $76.9(11.8)$ & $75.9(11.8)$ \\
\hline Height $(\mathrm{m})$ & $1.72(0.1)$ & $1.76(0.07)$ & $1.68(0.08)$ \\
\hline Body mass index (kg.m $\left.{ }^{-2}\right)$ & $25.8(3.1)$ & $24.8(2.6)$ & $26.8(3.3)$ \\
\hline $\begin{array}{l}\text { Reconstruction: Hamstring/patella tendon grafts n } \\
\text { (\%) }\end{array}$ & $15(48) / 16$ & $9(56) / 7(44)$ & $6(35) / 9(64)$ \\
\hline Meniscal repair: no/yes n (\%) & $23(74) / 8(26)$ & $13(81) / 3$ & $10(67) / 5$ \\
\hline Time since ACL injury (months) & & $(19)$ & $(33)$ \\
\hline Time since surgery (months) & $21(9-108)$ & $21(9-55)$ & $21(12-108)$ \\
\hline Time from ACL injury to surgery (months) & $16(6-53)$ & $17(6-44)$ & $16(7-53)$ \\
\hline Tegner activity scale: Preinjury (median, range) & $8(3-10)$ & $9(3-10)$ & $7(6-10)$ \\
\hline Tegner activity scale: Baseline (median, range) & $5(2-9)$ & $5(2-9)$ & $4(2-9)$ \\
\hline IKDC-SKF Baseline & $64.1(9.6)$ & $69.2(10.2)$ & $66.1(9.0)$ \\
\hline IKDC-SKF Follow-up & $73.7(11.5)$ & $71.3(13.2)$ & $76.5(8.9)$ \\
\hline Figures are numbers (Frequency), Mean (SD) or Medians (minimum & - maximum) & \\
\hline IKDC-SKF: International Knee Documentation Committee Subjective Knee Form & $6(1-11)$ & $8(1-89)$ \\
\hline
\end{tabular}

Due to the large rate of participants lost to follow-up for the biomechanical data (42\% of 31 participants), we compared the IKDC-SKF at baseline and at follow-up between those whose data were included in the results to those that were excluded or did not attend the second laboratory session. There was no statistically significant difference for IKDC-SKF between participants whose data were included in the RCT $(n=18 ; 68.1$ [9.3]) and those who were lost to follow-up for the biomechanical data $(n=13 ; 67.1$ [10.4], $p=0.775$ ). Similarly, there were no differences for the IKDC-SKF at follow-up (included: 73.3 [13.1]; lost to follow-up: 74.2 [8.9], $p=0.863$ ). 
The knee flexion angle trajectories of the unsleeved uninjured side, unsleeved and sleeved injured sides (Fig. 4a) differed across most of the stance phase (Fig. 4b). Those differences were explained mainly by differences during mid-phase stance (during which peak flexion occurs) when comparing the injured to the uninjured sides (Fig. 4c) and during the first third of the stance phase when comparing the injured side sleeved to the unsleeved conditions (Fig. 4e). Those differences were confirmed with post-hoc discrete variable analysis (Table 2): the (unsleeved) injured side had less peak flexion compared to the (unsleeved) uninjured side. When wearing the sleeve, the injured side flexion angle at initial contact and at its peak increased compared to not wearing the sleeve. On average, the total flexion excursion range during stance was less for the injured side compared to the uninjured side, and did not change when wearing a sleeve.

The knee external flexion moment trajectory of the unsleeved uninjured side, the unsleeved injured sides and sleeved injured sides (Fig. 5a) also differed across most of the stance phase (Fig. 5b). Those differences were explained by differences between the (unsleeved) uninjured and (unsleeved) injured sides from 5 to $80 \%$ of stance (Fig. 5 c). The post-hoc discrete variable analysis confirmed that the (unsleeved) injured knee had lower peak knee flexion moments compared to the uninjured side. However, discrete variable analysis contrasted to SPM when comparing injured side peak knee flexion moments while wearing the sleeve to not wearing the sleeve (Fig. 5e; Table 2). Wearing the sleeve, on average, increased the peak knee flexion moments compared to the unsleeved condition (Table 2).

Table 2

Immediate effects of wearing the sleeve: cross-over trial $(n=31)$

\begin{tabular}{|c|c|c|c|c|c|c|c|}
\hline & \multicolumn{2}{|l|}{$\begin{array}{l}\text { Unsleeved } \\
\text { Condition }\end{array}$} & \multirow{2}{*}{$\begin{array}{l}\text { Sleeved } \\
\text { Condition } \\
\begin{array}{l}\text { Injured } \\
\text { side }\end{array}\end{array}$} & \multicolumn{2}{|c|}{$\begin{array}{l}\text { Between side } \\
\text { comparison } \\
\text { (unsleeved) }\end{array}$} & \multicolumn{2}{|c|}{$\begin{array}{l}\text { Between condition } \\
\text { comparison, } \\
\text { injured side }\end{array}$} \\
\hline & $\begin{array}{l}\text { Uninjured } \\
\text { side }\end{array}$ & $\begin{array}{l}\text { Injured } \\
\text { side }\end{array}$ & & $\begin{array}{l}\text { Mean } \\
\text { Difference } \\
(95 \% \mathrm{Cl})\end{array}$ & $\begin{array}{l}\mathrm{p}- \\
\text { value }\end{array}$ & $\begin{array}{l}\text { Mean } \\
\text { Difference } \\
(95 \% \mathrm{Cl})\end{array}$ & $\begin{array}{l}\mathrm{p} \text { - } \\
\text { value }\end{array}$ \\
\hline $\begin{array}{l}\text { Knee flexion angle } \\
\text { at initial contact }\left(^{\circ}\right)\end{array}$ & $9.5(5.3)$ & $\begin{array}{l}11.0 \\
(4.9)\end{array}$ & $13.5(4.9)$ & $\begin{array}{l}1.4(-0.5 \\
3.3)\end{array}$ & 0.148 & $\begin{array}{l}2.7(1.3 \\
4.1)\end{array}$ & $\begin{array}{l}< \\
0.001\end{array}$ \\
\hline $\begin{array}{l}\text { Knee peak flexion } \\
\text { angle }\left({ }^{\circ}\right)\end{array}$ & $\begin{array}{l}46.2 \\
(6.4)\end{array}$ & $\begin{array}{l}42.5 \\
(5.7)\end{array}$ & $45.4(7.1)$ & $\begin{array}{l}-3.9(-5.7 \\
-2.0)\end{array}$ & $<001$ & $\begin{array}{l}3.0(1.2 \\
4.9)\end{array}$ & 0.001 \\
\hline $\begin{array}{l}\text { Knee flexion/ } \\
\text { extension } \\
\text { excursion }\left({ }^{\circ}\right)\end{array}$ & $\begin{array}{l}36.7 \\
(5.1)\end{array}$ & $\begin{array}{l}31.4 \\
(4.4)\end{array}$ & $31.9(6.7)$ & $\begin{array}{l}-5.3(-7.6 \\
-2.9)\end{array}$ & $\begin{array}{l}<.001 \\
0.001\end{array}$ & $\begin{array}{l}0.4(-1.3 \\
2.0)\end{array}$ & 0.673 \\
\hline $\begin{array}{l}\text { Knee peak external } \\
\text { flexion moment } \\
\text { (Nm/BW.ht) }\end{array}$ & $\begin{array}{l}0.114 \\
(0.029)\end{array}$ & $\begin{array}{l}0.087 \\
(0.028)\end{array}$ & $\begin{array}{l}0.093 \\
(0.030)\end{array}$ & $\begin{array}{l}-0.027 \\
(-0.037 \\
-0.017)\end{array}$ & $\begin{array}{l}<.001 \\
0.00\end{array}$ & $\begin{array}{l}0.007 \\
(0.002 \\
0.011)\end{array}$ & 0.005 \\
\hline $\begin{array}{l}\text { Stance duration } \\
\text { (ms) }\end{array}$ & 380 (79) & $\begin{array}{l}376 \\
(68)\end{array}$ & 395 (85) & $\begin{array}{l}-4(-31 \\
22)\end{array}$ & 0.751 & $19(-5,43)$ & 0.127 \\
\hline
\end{tabular}




\section{Temporal variable}

On average, stance duration did not differ between injured and uninjured sides, nor when comparing the sleeved to the unsleeved conditions for the injured sides (Table 2).

\section{Part 2: Randomised clinical trial}

Data of nine participants in each group could be included in the analysis (Sleeve Group: 5 women, 4 men; Control Group: 3 women, 6 men). Knee angle trajectories for the Sleeve Group and the Control Groups are shown in Fig. 6 at (a) baseline and (b) 6-week follow-up. The SPM analysis of the baseline to follow-up mean changes between groups showed significant differences from 10 to $75 \%$ of stance phase (Fig. $6 \mathrm{~d}$ ). The Sleeve Group knee trajectory showed larger changes than for the Control Group, leading to less knee flexion at follow-up. The difference is not reflected with discrete variables for flexion (initial contact, peak flexion and excursion, Table 3). However, the $95 \%$ confidence intervals for changes from baseline to follow-up for the Control group indicate that initial contact and peak knee flexion angles increased, on average by $3^{\circ}$, with no change for total excursion (Table 3 ). 
Table 3

Randomised Clinical Trial: Parameters of injured sides at baseline and follow-up, and between-group differences of changes from baseline to follow-up.

\begin{tabular}{|c|c|c|c|c|c|c|c|}
\hline & \multicolumn{2}{|c|}{$\begin{array}{l}\text { Control Group } \\
\text { Mean (SD) }\end{array}$} & \multicolumn{2}{|c|}{$\begin{array}{l}\text { Sleeve Group } \\
\text { Mean (SD) }\end{array}$} & \multicolumn{2}{|c|}{$\begin{array}{l}\text { Change score } \\
\text { group } \\
\text { Mean difference } \\
(95 \% \mathrm{Cl})\end{array}$} & \multirow{2}{*}{$\begin{array}{l}\begin{array}{l}\text { Between } \\
\text { group } \\
\text { difference }\end{array} \\
\text { p-value* }\end{array}$} \\
\hline & $B L$ & FU & BL & FU & Control & Sleeve & \\
\hline $\begin{array}{l}\text { Flexion angle at initial } \\
\text { contact }\left(\left(^{\circ}\right)\right.\end{array}$ & $\begin{array}{l}11.1 \\
(5.3)\end{array}$ & $\begin{array}{l}14.1 \\
(6.8)\end{array}$ & $\begin{array}{l}11.8 \\
(4.7)\end{array}$ & $\begin{array}{l}13.0 \\
(6.6)\end{array}$ & $\begin{array}{l}3.0 \\
(0.8 \\
5.1)\end{array}$ & $\begin{array}{l}1.1 \\
(-1.5 \\
3.7)\end{array}$ & 0.310 \\
\hline Peak flexion angle $\left(^{\circ}\right)$ & $\begin{array}{l}41.2 \\
(5.9)\end{array}$ & $\begin{array}{l}43.9 \\
(6.5)\end{array}$ & $\begin{array}{l}43.2 \\
(5.6)\end{array}$ & $\begin{array}{l}40.0 \\
(8.0)\end{array}$ & $\begin{array}{l}2.8 \\
(0.1 \\
5.5)\end{array}$ & $\begin{array}{l}-3.2 \\
(-8.8 \\
2.5)\end{array}$ & 0.058 \\
\hline $\begin{array}{l}\text { Flexion/extension } \\
\text { Excursion }\left(^{\circ}\right)\end{array}$ & $\begin{array}{l}30.0 \\
(2.9)\end{array}$ & $\begin{array}{l}29.8 \\
(4.2)\end{array}$ & $\begin{array}{l}31.3 \\
(5.3)\end{array}$ & $\begin{array}{l}27.0 \\
(7.4)\end{array}$ & $\begin{array}{l}-0.2 \\
(-3.1 \\
2.7)\end{array}$ & $\begin{array}{l}-4.3 \\
(-11.1 \\
2.4)\end{array}$ & 0.627 \\
\hline $\begin{array}{l}\text { Peak external flexion } \\
\text { moment }\left(\mathrm{Nm} / \mathrm{BW}^{\star} \mathrm{HT}\right)\end{array}$ & $\begin{array}{l}0.086 \\
(0.031)\end{array}$ & $\begin{array}{l}0.097 \\
(0.027)\end{array}$ & $\begin{array}{l}0.081 \\
(0.034)\end{array}$ & $\begin{array}{l}0.081 \\
(0.026)\end{array}$ & $\begin{array}{l}0.011 \\
(-0.015 \\
0.036)\end{array}$ & $\begin{array}{l}0.000 \\
(-0.025 \\
0.025)\end{array}$ & 0.402 \\
\hline Stance duration (ms) & $\begin{array}{l}344 \\
(56)\end{array}$ & $\begin{array}{l}346 \\
(65)\end{array}$ & $\begin{array}{l}402 \\
(89)\end{array}$ & $\begin{array}{l}313 \\
(49)\end{array}$ & $\begin{array}{l}2(-35 \\
38)\end{array}$ & $\begin{array}{l}-89 \\
(-153 \\
-24)\end{array}$ & 0.013 \\
\hline
\end{tabular}

Knee external flexion moment trajectories for the Sleeve Group and Control Group are shown in Fig. 7 at (a) baseline and (b) 6-week follow-up. The SPM analysis of the baseline to follow-up mean changes showed no between-group differences during stance phase (Fig. 7d), also reflected in lack of differences for discrete peak flexion moments (Table 3).

\section{Temporal variable}

Stance duration changed to a greater extent for the Sleeve Group than the Control Group from baseline to follow-up (Table 3). For the stance group, the average duration decreased by $22 \%$.

\section{Discussion}

We determined immediate and six-week effects of wearing a knee sleeve on knee kinematics and kinetics in participants with an ACL reconstruction during a sub-maximal step-down-hop task. When unsleeved, 
the injured side had lower external knee flexion moments and flexion angles during stance of the stepdown hop compared to the uninjured side. When wearing the sleeve on the injured side, knee flexion increased to similar magnitudes as the uninjured side, particularly within the first $40 \%$ of stance. The knee flexion moments were lower for the injured compared to the uninjured sides, and remained unchanged in the immediate intervention with the knee sleeve. Thus, the first research hypothesis $(\mathrm{H} 1)$ was accepted for increased knee flexion angles when wearing the sleeve, but not for the flexion moments based on the SPM analysis. Following the six-week period, within-participant changes from baseline to follow-up differed between the two groups: the Sleeve Group appeared to have less knee flexion at follow-up, whereas the Control group who had slightly greater (not significant) knee flexion. These differences applied to most of the stance phase. Thus, we found no support for our second research hypothesis $(\mathrm{H} 2)$, that the Sleeve Group would have increased knee flexion angles and moments at follow-up. However, the analysis of stance duration suggests significantly different responses between the groups: while the Control group had no change in stance duration, the Sleeve group had a shorter stance duration at followup, with the time decreasing by $22 \%$ compared to the baseline duration. The Sleeve Group thus performed the task at a faster speed, possibly indicating overall enhanced performance. Thus, we found support for our third research hypothesis of decreased stance duration for the Sleeve Group at follow-up.

\section{Kinematic responses: knee flexion angles}

Reduced knee flexion angles during jump landing have been explored extensively in terms of risk for ACL rupture and as outcomes of such injury and reconstruction [26]. Knee flexion contributes to absorption of impact forces when landing, along with hip flexion and ankle dorsiflexion [27]. Decreased angles (thus a more extended knee) are suggested to indicate 'stiffer' knee landing patterns, and are proposed to expose the knee joint to higher forces $[13,26]$. Programmes to minimise risk have focussed on re-training landing with 'softer' knees, that is, increasing peak knee flexion on landing $[12,13]$. Our SPM analysis showed that when compared to the contralateral side, participants landed with reduced knee flexion at 35 to $65 \%$ of the stance phase when unsleeved. Peak knee flexion occurs during that period. When wearing the sleeve, the knee flexion of the injured side increased significantly during the first $40 \%$ of stance phase, corresponding to roughly the first $180 \mathrm{~ms}$. ACL ruptures are likely to occur in the first $50 \mathrm{~ms}$ following landing [28], thus, we can speculate that enhanced flexion during that phase when wearing the sleeve may have a protective effect.

A clinically meaningful increase in knee flexion angles has to date not been defined, and may vary throughout the recovery phase. These most likely depend on the overall excursion during the specific task. Previous laboratory-based studies showed that wearing a sleeve can enhance knee flexion angles and influence frontal plane biomechanics during walking in participants with knee osteoarthritis $[6,7]$. Knee flexion angles, however, increased at an average of $1.2^{\circ}[6]$, and knee adduction angles decreased by $1.9^{\circ}$ at initial ground contact and at $1.5^{\circ}$ at peak [7]. Ericksen, Thomas, Gribble, Doebel and Pietrosimone [29] reported an increase of $10^{\circ}$ flexion when providing real-time feedback to healthy women during double-leg jump landing. However, using the same double-leg jump with a mixed group knee-healthy men and women, Welling, Benjaminse, Gokeler and Otten [13] found differences of around 
$2^{\circ}$, on average, with provision of various forms of verbal feedback. Those studies entailed maximal vertical jump, leading to peak knee flexion ranging between approximately 60 to $70^{\circ}[13,29]$. In comparison, we used a sub-maximal horizontal hop with an average of $46^{\circ}$ peak flexion for the uninjured side. Yet, we found a mean difference between the injured and uninjured side of $4^{\circ}$, and wearing the sleeve increased flexion angles at initial contact and at its peak by $3^{\circ}$. Our test-retest reliability data indicates a standard error of measurement for peak knee flexion angles to be $1.5^{\circ}$, and a smallest detectable difference of $4^{\circ}$ (Supplementary file). Participants did not focus on their knee movement patterns on landing, instead were prompted to step down and jump forwards 'as fast as possible'. Despite their attention not being on the knee position or movement, at baseline, the injured side average peak flexion increased when wearing the sleeve. As the flexion at initial contact also increased in the sleeved condition, the resulting flexion excursion range did not change. Thus, wearing a knee sleeve could potentially be used as an adjunct to jump-landing training. Mechanisms underlying those changes are likely due to the tactile sensation of the knee sleeve improving proprioception or awareness of the knee, leading to greater confidence with undertaking the physical task [6-8].

\section{Kinetic responses: external knee flexion moments}

The SPM analysis showed that external knee flexion moments were lower for the injured side compared to the uninjured side. The discrete variable analysis showed a between-side $24 \%$ difference for peak flexion moments. Such reduced peak knee flexion moments during various types of jump when comparing ACL-reconstructed knees with healthy controls and compared to the contralateral limb have also been reported previously [26]. While the SPM showed no significant differences for the injured side flexion moment trajectory between sleeved and unsleeved, the discrete variable analysis suggests a significant increase of 7\%. Differences in the results between SPM and discrete variable analyses may relate to the fact that the peak moments do not necessarily occur at the same time point during stance phase for each participant. Comparing time-based trajectories (as with the SPM) ensures magnitudes at equivalent events are compared, whereas discrete variables introduce potential covariation with event timing. Despite the small increase for the injured side flexion moment, it remained substantially lower compared to the uninjured (unsleeved) side. Furthermore, our test-retest reliability data with 10 kneehealthy participants suggests a standard error of measurement to be $6 \%$ for peak external knee flexion moments. Thus, we cannot rule out that the differences observed for knee flexion moments between the sleeved and unsleeved conditions may be due to measurement error.

\section{Stance duration}

Stance duration did not differ significantly between the injured and the uninjured sides, nor were differences observed for the injured side when wearing the sleeve in the cross-over study. At six-week follow-up, the change scores for stance duration were different between the two experimental groups: while the Control group showed no difference in duration, the Sleeve group had shorter duration (exceeding the smallest detectable difference, Supplementary file). 
The shorter stance duration at follow-up for the Sleeve Group, with no changes in knee flexion angles and moments may indicate enhanced ability to attenuate landing forces and to generate power for the forward hop. Participants were instructed to 'jump forward as fast as possible', thus shorter durations indicate an improvement. Faster movement without changes in knee flexion moments (as observed for the Sleeve Group) must result in no change in flexion angle. In our earlier study [5], we reported that the Sleeve Group had higher self-reported physical activity than the Control group, but no other betweengroup differences were found for self-reported function (IKDC-SKF), hop distance and thigh muscle strength. Increased physical activity levels reported by participants of the Sleeve Group may have improved knee function, potentially leading to enhanced power generation associated with improved movement efficiency. In combination, improved physical activity level and sensorimotor effects of the knee sleeve may have improved confidence in the knee [6-8], evident in faster action during the individual-specific standardised forward hop.

We explored the IKDC-SKF, isokinetic thigh muscle strength, the maximum horizontal single-leg hop [5] and biomechanical measures during the sub-maximal step-down hop. The stance duration of the latter was the only significant change for the Sleeve Group from baseline to follow-up. Thus, it appears that the only significant change was related to movement efficiency, in the absence of change in self-reported function and the maximum physical performance tests. Further analyses of knee power during the task and of the ground reaction forces are warranted.

\section{Methodological considerations}

This investigation was a secondary analysis of the influence of wearing a knee sleeve on various movement-related variables. The study was affected by the 6-week COVID-19 lockdown of 2020 in New Zealand, losing 8 participants for the laboratory follow-up session. Due to time limits for this study, we were not able to recruit sufficient participants to meet the planned sample size of 16 per group. Our results may thus reflect a Type 2 error for the 6-week effects of wearing a sleeve.

Here we analyse movement patterns only in the sagittal plane as we found low reliability for knee kinematics and kinetics in the transvers and frontal plane during the step-down task. We also did not explore possible compensatory movements at the trunk, hip, ankle and foot. Lastly, movement patterns during landing differ between men and women which may have confounded our results. A larger sample size would be needed to explore whether men and women respond differently to wearing a knee sleeve.

A strength of our analysis is that our primary analysis was based on SPM to explore differences between trajectories across the entire stance phase. We report post hoc discrete variable analysis to allow comparison with other studies. A limitation of discrete variable analyses is that they only report one timepoint in the entire movement. Furthermore, discrete variable analysis ultimately leads to multiple comparisons, with the risk of Type I errors. We report the p-values, as well as mean differences (and 95\% Cls) between conditions (Part 1) and groups (Part 2) to allow the reader to interpret the outcomes.

\section{Conclusions}


In a group of 31 participants with ACL reconstruction, knee flexion angles increased during the first $40 \%$ of the stance phase during a sub-maximal step-down hop task when wearing a knee sleeve compared to the unsleeved condition. There were no significant differences for external knee flexion moments between the two conditions. Wearing the sleeve for 6 weeks, for a minimum of 1 hour per day, did not lead to increased knee flexion angles or external knee flexion moments compared to the control group who did not receive that sleeve. However, stance duration decreased significantly for the Sleeve Group compared to the Control Group, potentially indicating faster or enhanced physical performance during that task. The knee sleeve might be used as an adjunct to rehabilitation to increase knee flexion angles. Prescription of such sleeve should be based on individualised assessment of immediate responses and over a defined time period.

\section{Declarations}

\section{Ethics approval and content to participate}

Ethics approval was granted by the Health \& Disability Ethics Committee, New Zealand, Reference $18 / C E N / 93$, dated $5^{\text {th }}$ June 2018 , amended on $27^{\text {th }}$ August 2019 and $4^{\text {th }}$ October 2019. All participants provided written informed consent to participate. All methods were undertaken in accordance with the Declaration of Helsinki.

\section{Consent for publication}

The authors have obtained the participants' written informed consent for print and electronic publication of this research. Written informed consent was also provided by the participant for the publication of his image in Figure 2.

\section{Availability of data and materials}

The datasets used and/or analysed during the current study are available from the corresponding author on reasonable request.

\section{Competing interests}

Bauerfeind AG funded this study. There are no patents, products in development or marketed products to declare. The authors declare they have no competing interests.

\section{Funding}

Funding and material (knee sleeves) were provided by Bauerfeind AG (Triebeser Straße, 07937 Zeulenroda-Triebes, Germany. The Funder was not involved in participant recruitment, data collection, processing and analysis, and interpretation of results, and in no way influenced experimental procedures or report preparation. The funding covered the overheads for the Biomechanics laboratory, laboratory 
technician, administrative support, research assistant, contribution towards participant transport costs, and staff-related overheads.

\section{Authors' contributions}

GS and NH instigated this research project; GS, PL, TP, SK and NH contributed to the research design; GS provided necessary administrative support; GS provided participant recruitment; GS and AP collected and processed data; TP, GS, and a colleague at the University of Otago Biostatistics Centre contributed to the statistical methodology. GS, PL, TP, SK, PN and NH assisted with the initial construction and direction of the discussion, and developing the multiple iterations of the manuscript. All authors reviewed and provided constructive advice and critique to the editing process, read and approved the final report.

\section{Acknowledgements}

We thank the Biostatistics Centre, Division of Health Sciences, University of Otago, Dunedin, for performing the statistical analyses of the outcomes of the trial. We also thank David Jackson, Dr Sarah Ward and Dr Mandeep Kaur for their assistance for data collection and processing.

\section{References}

1. van Melick N, van Cingel REH, Brooijmans F, Neeter C, van Tienen T, Hullegie W, Nijhuis-van der Sanden MWG: Evidence-based clinical practice update: practice guidelines for anterior cruciate ligament rehabilitation based on a systematic review and multidisciplinary consensus. Br J Sports Med 2016, 50(24):1506.

2. Mahood C, Perry M, Gallagher P, Sole G: Chaos and confusion with confidence: Managing fear of ReInjury after anterior cruciate ligament reconstruction. Physical Therapy in Sport 2020, 45:145-54.

3. Martinez-Calderon J, Flores-Cortes M, Morales-Asencio JM, Fernandez-Sanchez M, Luque-Suarez A: Which interventions enhance pain self-efficacy in people with chronic musculoskeletal pain? A systematic review with meta-analysis of randomized controlled trials, including over 12000 participants. J Orthop Sports Phys Ther 2020, 50(8):418-30.

4. Ardern CL, Taylor NF, Feller JA, Webster KE: Fear of re-injury in people who have returned to sport following anterior cruciate ligament reconstruction surgery. J Sci Med Sport 2012, 15(6):488-95.

5. Sole G, Lamb P, Pataky T, Klima S, Navarre P, Hammer N: Immediate and 6-week effects of wearing a knee sleeve following anterior cruciate ligament reconstruction: a cross-over laboratory and randomised clinical trial. BMC Musculoskelet Disord 2021, 22(1).

6. Collins A, Blackburn T, Olcott C, Jordan JM, Yu B, Weinhold P: A kinetic and kinematic analysis of the effect of stochastic resonance electrical stimulation and knee sleeve during gait in osteoarthritis of the knee. J Appl Biomech 2014, 30(1):104-12.

7. Schween R, Gehring D, Gollhofer A: Immediate effects of an elastic knee sleeve on frontal plane gait biomechanics in knee osteoarthritis. PLoS One 2015, 10(1):e0115782. 
8. Van Tiggelen D, Coorevits P, Witvrouw E: The use of a neoprene knee sleeve to compensate the deficit in knee joint position sense caused by muscle fatigue. Scand J Med Sci Sports 2008, 18:62-6.

9. Nagelli C, Di Stasi S, Tatarski R, Chen A, Wordeman S, Hoffman J, Hewett TE: Neuromuscular training improves self-reported function and single-leg landing hip biomechanics in athletes after anterior cruciate ligament Reconstruction. Orthopaed J Sports Med 2020, 8(10):2325967120959347.

10. Neilson V, Ward S, Hume P, Lewis G, McDaid A: Effects of augmented feedback on training jump landing tasks for ACL injury prevention: A systematic review and meta-analysis. PhysTher Sport 2019, 39:126-135.

11. Larson D, Nathan Vannatta C, Rutherford D, Kernozek TW: Kinetic changes associated with extended knee landings following anterior cruciate ligament reconstruction in females. Phys Ther Sport 202152:180-8.

12. Webster KE, Hewett TE: Meta-analysis of meta-analyses of anterior cruciate ligament injury reduction training programs. J Orthopaed Res 2018, 36(10):2696-208.

13. Welling W, Benjaminse A, Gokeler A, Otten B: Retention of movement technique: implications for primary prevention of ACL injuries. Int J Sports Phys Ther 2017, 12(6):908-90.

14. Jeon H, Krysak S, Pfeiffer SJ, Thomas AC: Plyometrics did not improve jump-landing biomechanics in individuals with a history of anterior cruciate ligament reconstruction: a randomized controlled trial. Int J Athl Ther Train 2021: doi:10.1123/ijatt.2020-0072.

15. Schulz KF, Altman DG, Moher D, the CG: CONSORT 2010 Statement: updated guidelines for reporting parallel group randomised trials. Trials 2010, 11(1):32.

16. Irrgang JJ, Anderson AF, Boland AL, Harner CD, Kurosaka M, Neyret P, Richmond JC, Shelborne KD: Development and validation of the international knee documentation committee subjective knee form. Am J Sports Med 2001, 29(5):600-13.

17. Tegner Y, Lysholm J: Rating systems in the evaluation of knee ligament injuries. Clin Orthop Relat Res 1985, 198:43-9.

18. van Meer BL, Meuffels DE, Vissers MM, Bierma-Zeinstra SMA, Verhaar JAN, Terwee CB, Reijman M: Knee Injury and Osteoarthritis Outcome Score or International Knee Documentation Committee Subjective Knee Form: Which questionnaire is most useful to monitor patients with an anterior cruciate ligament rupture in the short term? Arthroscopy 2013, 29(4):701-15.

19. Lefevre N, Klouche S, Mirouse G, Herman S, Gerometta A, Bohu Y: Return to sport after primary and revision anterior cruciate ligament reconstruction: A prospective comparative study of 552 patients from the FAST Cohort. Am J Sports Med 2017, 45:34-41.

20. Wu G, Siegler S, Allard P, Kirtley C, Leardini A, Rosenbaum D, Whittle M, D'Lima DD, Cristofolini L, Witte $\mathrm{H}$ et al: ISB recommendation on definitions of joint coordinate system of various joints for the reporting of human joint motion-part l: ankle, hip, and spine. J Biomech 2002, 35(4):543-48.

21. Kristianslund E, Krosshaug T: Comparison of drop jumps and sport-specific sidestep cutting: implications for anterior cruciate ligament injury risk screening. Am J Sports Med 2013, 41(3):684-8. 
22. Begon M, Monnet T, Lacouture P: Effects of movement for estimating the hip joint centre. Gait Posture 2007, 25(3):353-9.

23. Cappozzo A, Cappello A, Croce UD, Pensalfini F: Surface-marker cluster design criteria for 3-D bone movement reconstruction. IEEE Trans Biomed Eng 1997, 44(12):1165-74.

24. Friston KJ, Holmes AP, Poline JB, Grasby PJ, Williams SC, Frackowiak RS, Turner R: Analysis of fMRI time-series revisited. Neuroimage 1995, 2(1):45-53.

25. Pataky TC, Robinson MA, Vanrenterghem J: Vector field statistical analysis of kinematic and force trajectories. J Biomech 2013, 46(14):2394-401.

26. Johnston PT, McClelland JA, Webster KE: Lower limb biomechanics during single-leg landings following anterior cruciate ligament reconstruction: A systematic review and meta-analysis. Sports Med 2018, 48(9):2103-26.

27. Podraza JT, White SC: Effect of knee flexion angle on ground reaction forces, knee moments and muscle co-contraction during an impact-like deceleration landing: Implications for the non-contact mechanism of ACL injury. Knee 2010, 17(4):291-5.

28. Carlson VR, Sheehan FT, Boden BP: Video analysis of anterior cruciate ligament (ACL) injuries. JBJS Reviews 2016, 4(11):1.

29. Ericksen HM, Thomas AC, Gribble PA, Doebel SC, Pietrosimone BG: Immediate effects of real-time feedback on jump-landing kinematics. J Orthop Sports Phys Ther 2015, 45(2):112-8.

\section{Figures}




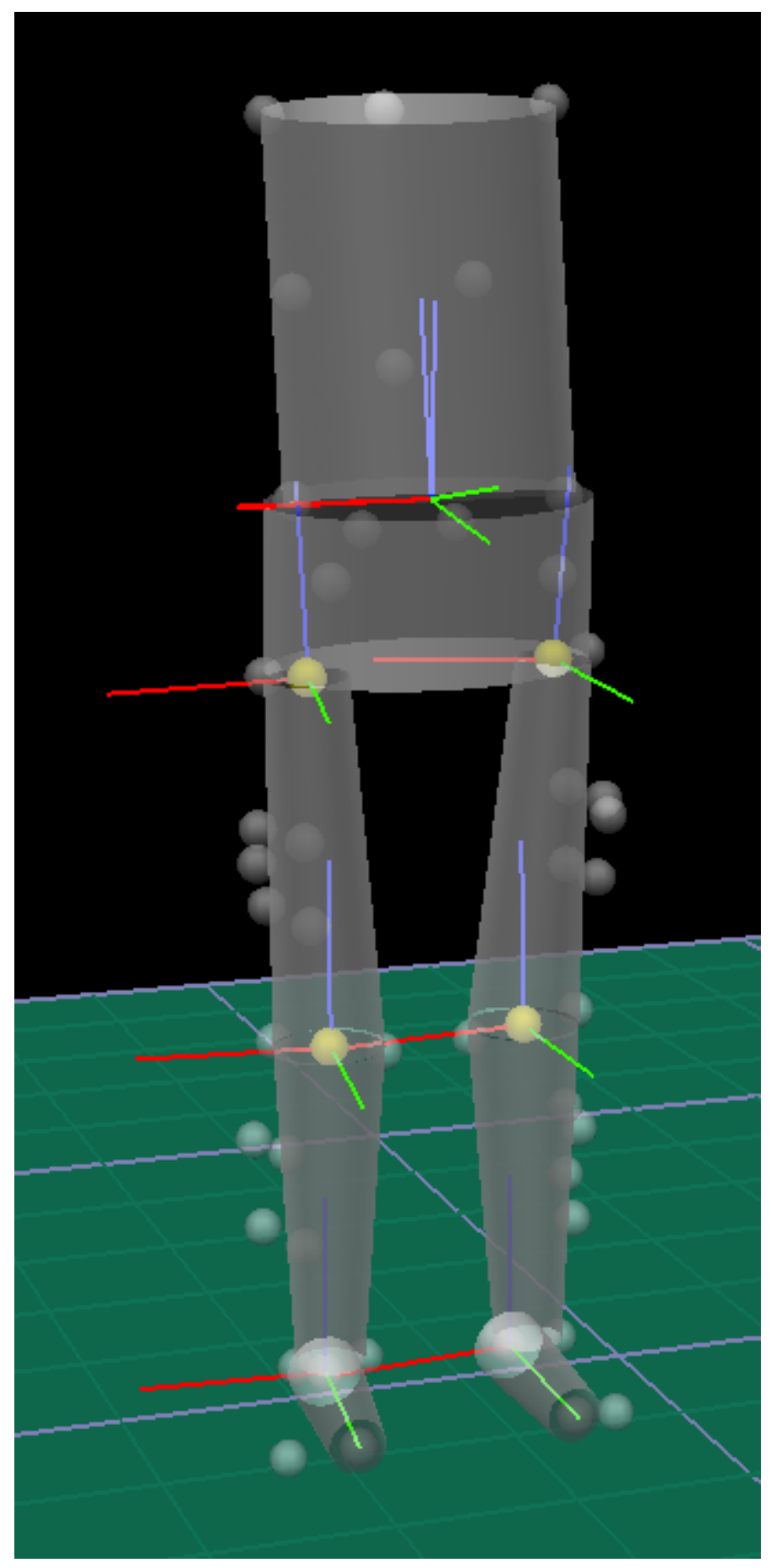

\section{Figure 1}

Placement of reflective markers: acromia, C7, T10, inferior scapula angles, posterior iliac spines, iliac crests, greater trochanter, medial and lateral knee, medial and lateral malleoli, a 5-marker cluster on the later thigh, a 4-marker cluster on the lateral shin, heel of shoe, metatarsal head 1, base of metatarsal 5 . 


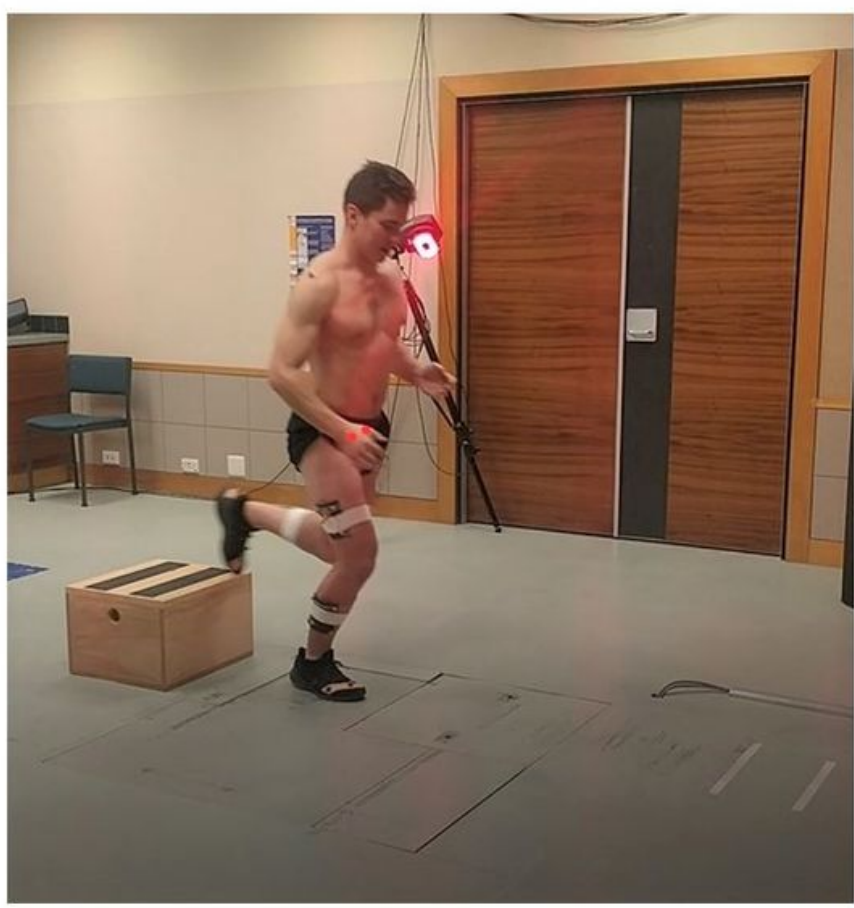

(a)

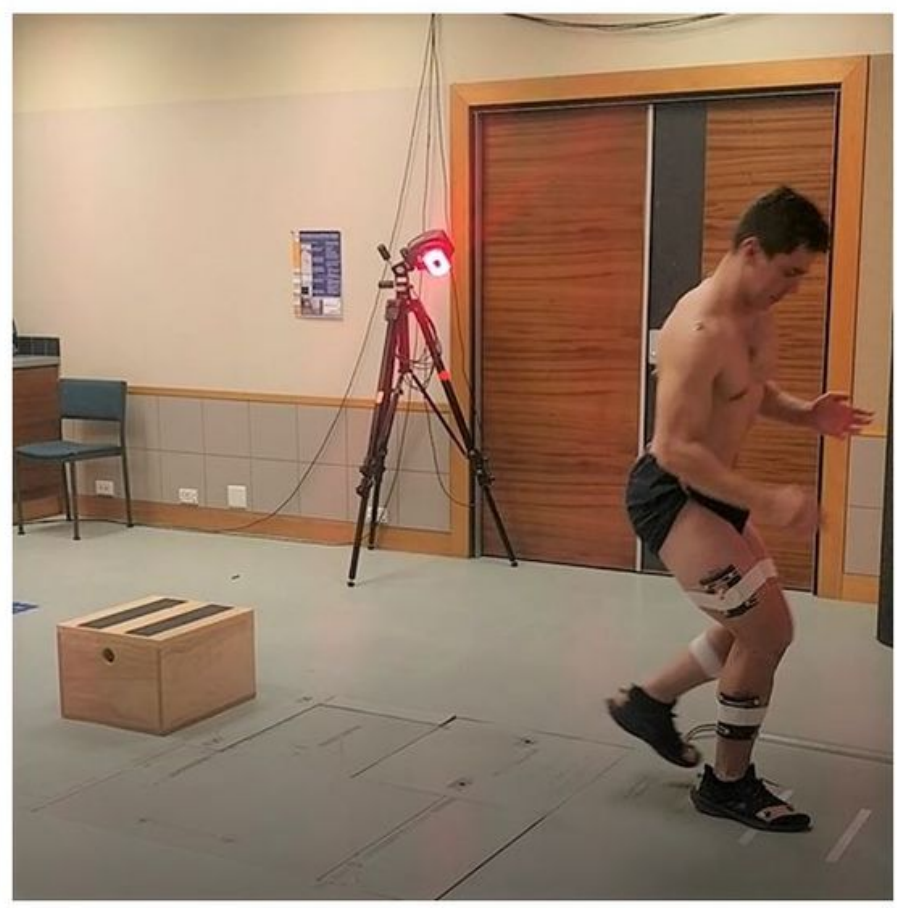

(b)

\section{Figure 2}

The participant is performing the step-down hop with the right side. (a) Stance phase on the floorembedded force plate; (b) hop landing at $60-70 \%$ of maximum horizontal jump distance, marked by two strips on the floor. 
Assessed for eligibility

Otago advertising: $n=50$

Trialfacts: $n=78$

(Total: $n=128$ )

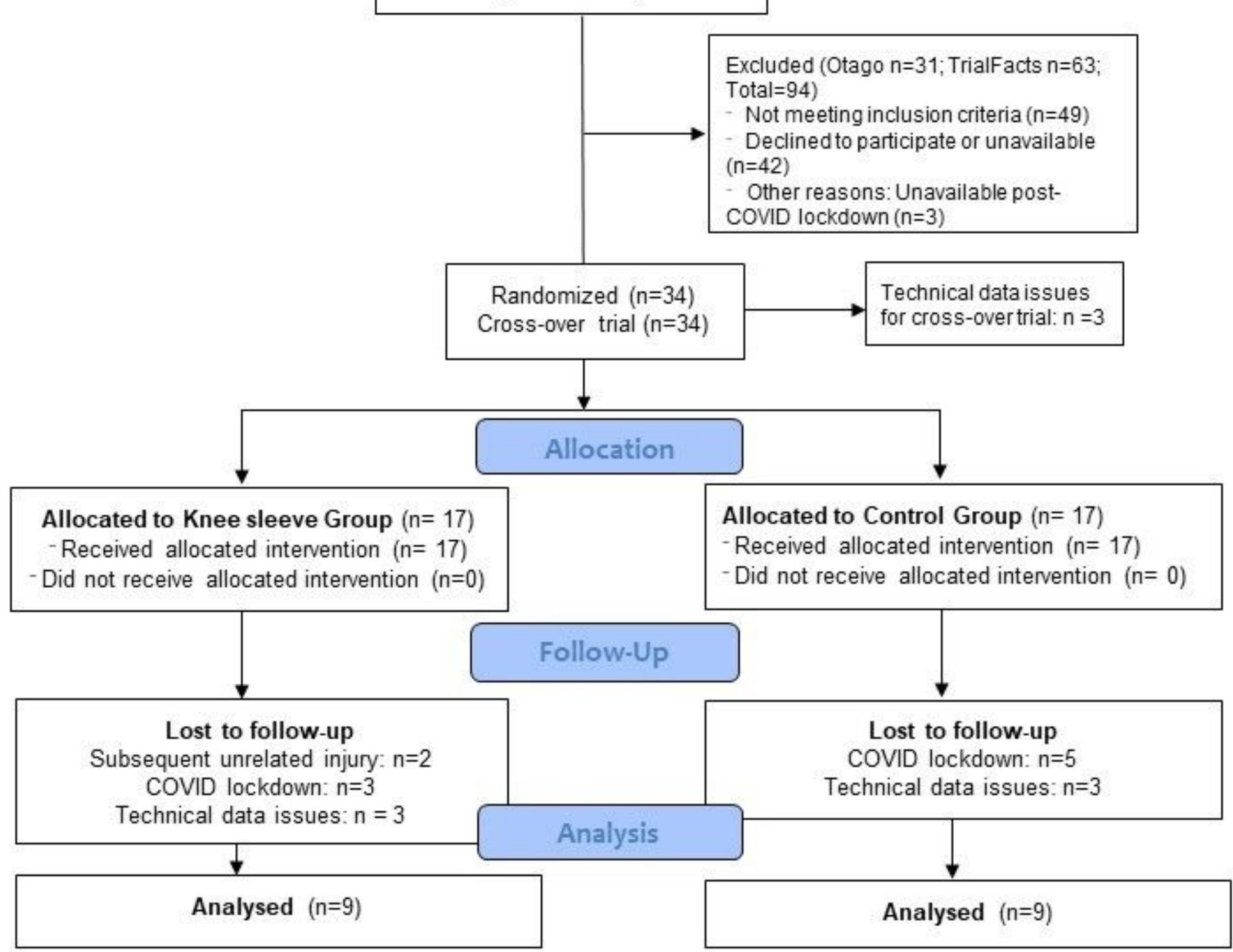

\section{Figure 3}

\section{Flowchart of participant recruitment, allocation and follow-up.}

* Participants were lost to the laboratory-based follow-up data collection due to the COVID-19 lockdown in March/April 2020. IKDC-SKF: International Knee Documentation Committee Subjective Knee Form 

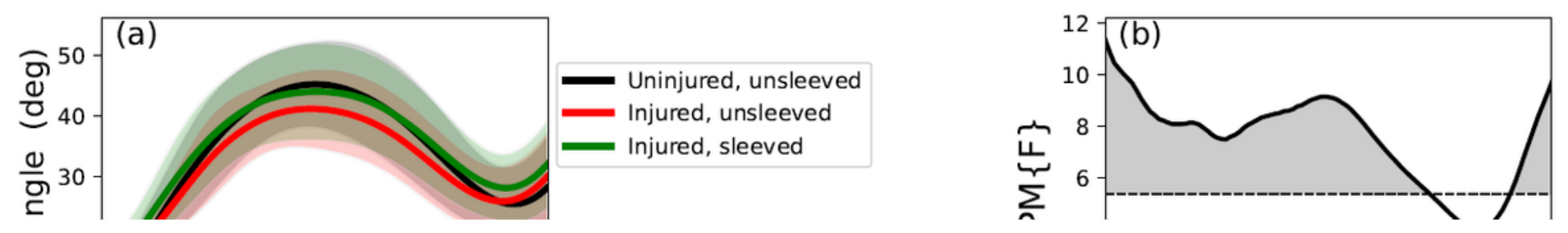

\section{Figure 4}

Statistical Parametric Mapping of knee flexion angle (degrees) trajectory during the stance phase of the step-down-and-hop task: (a): mean and individual participants' time series for flexion angle trajectories for uninjured sides, and unsleeved and sleeved injured sides; (b): ANOVA on the knee angle trajectory; post-hoc analyses: (c): uninjured side versus unsleeved injured; (d): uninjured versus sleeved injured; (e): injured side, sleeved versus unsleeved conditions. 

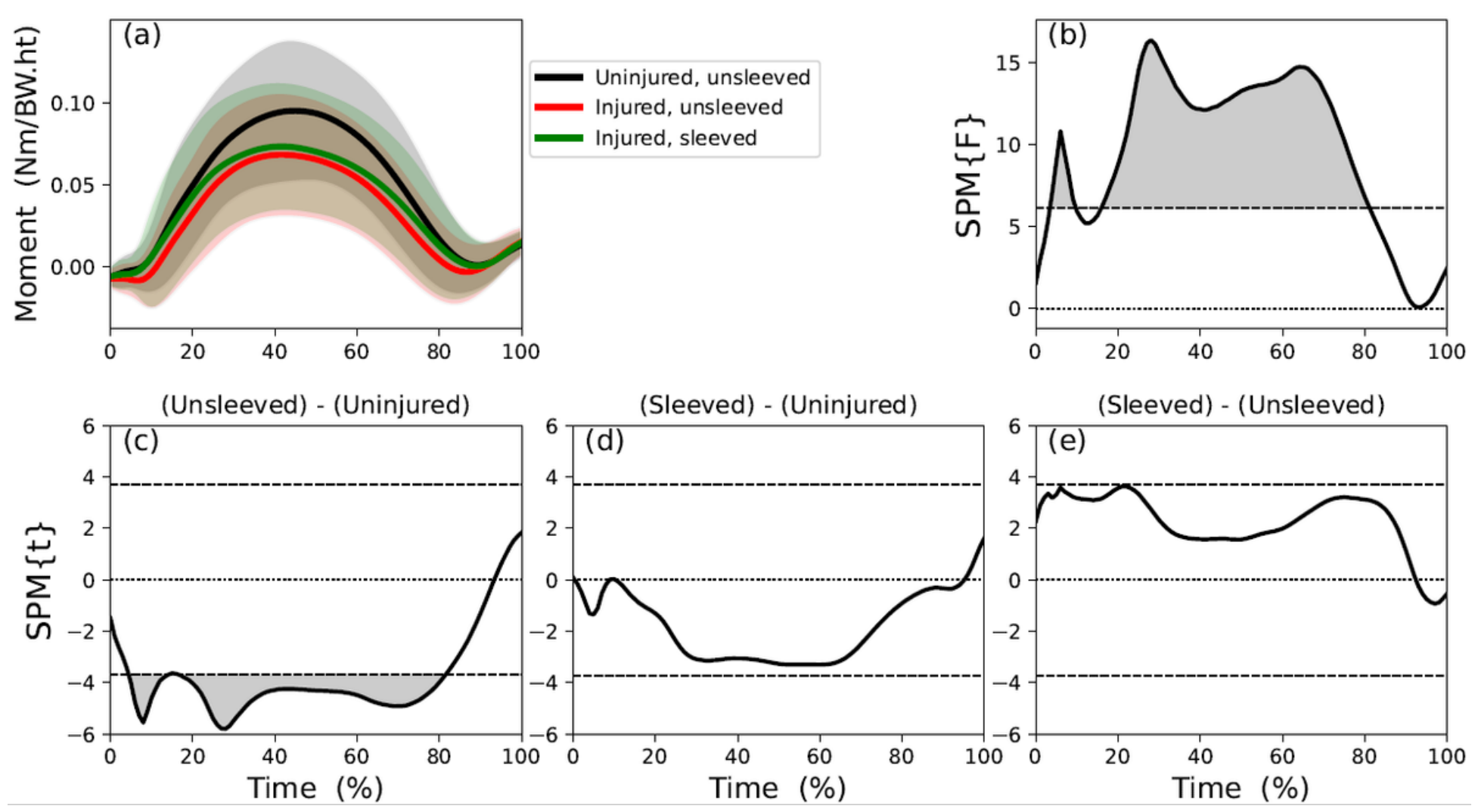

\section{Figure 5}

Statistical Parametric Mapping of external knee flexion moment (Nm/BW.ht) trajectory during the stance phase of the step-down-and-hop task: (a): mean and individual participants' time series for external knee flexion moment trajectories for uninjured sides, and unsleeved and sleeved injured sides; (b): ANOVA on the knee flexion moment trajectory; Post-hoc analyses: (c): uninjured side versus unsleeved injured; (d): uninjured versus sleeved injured; (e): injured side, sleeved versus unsleeved conditions. 

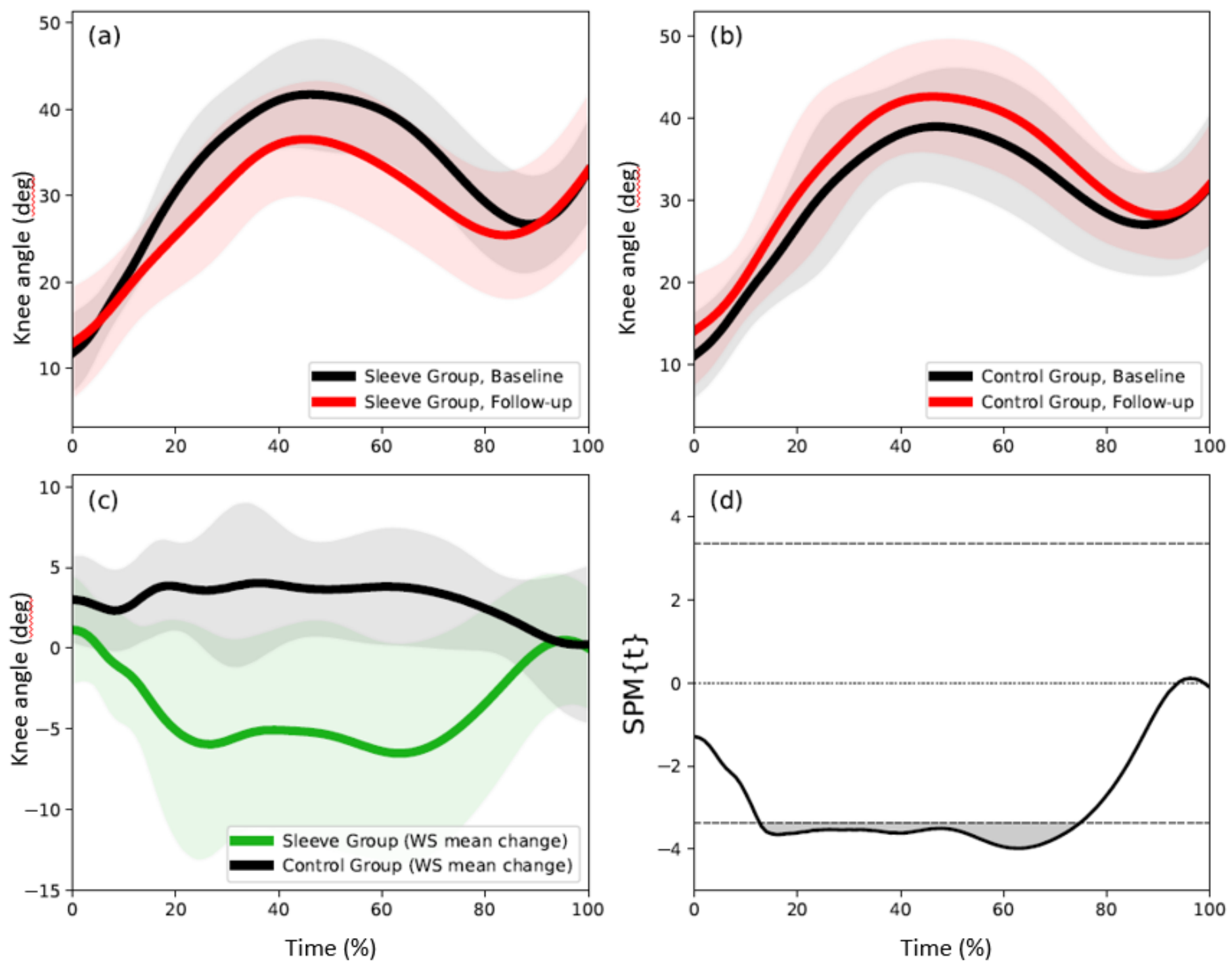

Figure 6

Injured side knee flexion angle trajectory (degrees) during stance of the step-down-hop task for the (a) Sleeve Group and (b) Control Groups; (c) within-participant mean differences between baseline and follow-up for the Sleeve and the Control Groups; (d) between-group SPM analysis (independent t tests) of the baseline to follow-up within-participant mean changes. (WS: within-subject) 

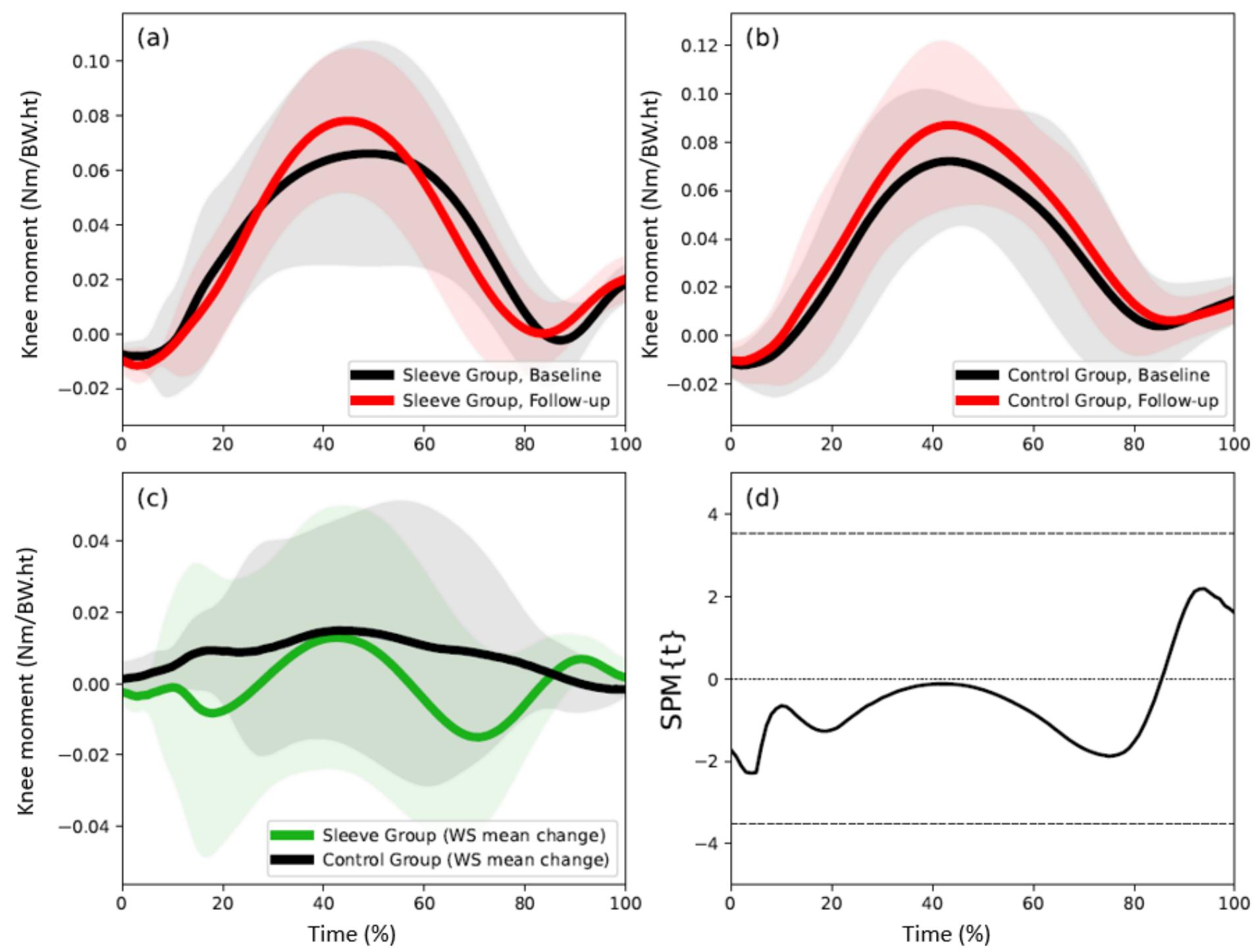

Figure 7

Injured side knee external flexion moment (Nm/BW.ht) trajectories during stance of the step-down-hop task for the (a) intervention and (b) control groups; (c) within-participant mean differences between baseline and follow-up for the intervention and the control groups; (d) between-group SPM analysis (independent $t$ tests) of the baseline to follow-up mean changes. (WS: within-subject)

\section{Supplementary Files}

This is a list of supplementary files associated with this preprint. Click to download.

- Supplementaryfile.docx 